\title{
Analysis of Remifentanil-Based Fast-Track Anesthesia Combined with Dexmedetomidine for Transthoracic Device Closure of Atrial Septal Defect in Pediatric Patients
}

\author{
Ling-Shan Yu, MM, ${ }^{1,2}$ Yu-Qing Lei, MM, Jian-Feng Liu, MM, Jing Wang, MD,${ }^{1}$ Hua Cao, MD,${ }^{1}$ \\ Zeng-Chun Wang, MD, ${ }^{1,2}$ Qiang Chen, MD $^{1,2}$ \\ ${ }^{1}$ Department of Cardiac Surgery, Fujian Maternity and Child Health Hospital, Affiliated Hospital of Fujian Medical University, \\ Fuzhou, China; ${ }^{2}$ Department of Cardiovascular Surgery, Union Hospital, Fujian Medical University, Fuzhou, China
}

\section{ABSTRACT}

Background: To investigate the safety and efficacy of remifentanil combined with dexmedetomidine in fast-track cardiac anesthesia (FTCA) for transthoracic device closure of atrial septal defect (ASD) in pediatric patients.

Methods: A retrospective analysis was performed on 61 cases of children undergoing ASD closure through a small thoracic incision from January 2018 to January 2020. According to whether FTCA was administered, they were divided into group $\mathrm{F}$ (fast-track anesthesia, $\mathrm{n}=31$ ) and group $\mathrm{R}$ (routine anesthesia, $\mathrm{n}=30$ ).

Results: There was no significant difference in general preoperative data, perioperative hemodynamics, or postoperative pain scores between the 2 groups $(\mathrm{P}>.05)$. The postoperative sedation score of group $\mathrm{F}$ was higher than that of group R 1 and 4 hours after extubation. Meanwhile, duration of mechanical ventilation and length of postoperative intensive care unit (ICU) stay of group F were significantly shorter than those of group $\mathrm{R}(\mathrm{P}<.05)$. No serious anesthesia-related complications occurred.

Conclusion: Remifentanil combined with dexmedetomidine in FTCA for transthoracic device closure of ASD in pediatric patients is safe and effective, is worthy of clinical promotion, and can benefit more children.

\section{INTRODUCTION}

With the development of minimally invasive cardiac surgery, patients can recover quickly, have fewer complications,

Z-C. Wang and Q. Chen share senior authorship.

Received October 26, 2020; accepted November 16, 2020.

Correspondence: Qiang Chen, MD, Department of Cardiac Surgery, Fujian Maternity and Child Health Hospital, Affiliated Hospital of Fujian Medical University, Fuzhou, China and Department of Cardiovascular Surgery, Union Hospital, Fujian Medical University, Fuzhou, China (e-mail: chenqiang2228@163.com). and spend less time in the hospital, necessitating a higher requirement for anesthesia methods applied in minimally invasive cardiac surgery to stop mechanical ventilation early after surgery, to shorten intensive care unit (ICU) stay, and to reduce anesthesia-related complications. Verrier et al [1995] first proposed the concept of "fast-track surgery" in 1993, and fast-track anesthesia has become an essential part of fast-track surgery. Fast-track cardiac anesthesia (FTCA) involves choosing appropriate anesthesia methods and drugs, striving to immediately (or as soon as possible) remove endotracheal intubation (within 6 hours after the surgery), reducing the length of postoperative ICU and hospital stays, and ultimately reducing medical costs [Mittnacht 2010]. At present, the application of FTCA in ultrasound-guided transthoracic device closure of atrial septal defect (ASD) in pediatric patients is still rare [Huang 2019]. Here, we report on our experience in this field.

\section{METHODS}

This study was approved by our university's ethics committee and adhered to the Declaration of Helsinki. In addition, written informed consent was obtained from the patients' parents.

A total of 61 cases undergoing ASD closure through a small thoracic incision were recorded from January 2018 to January 2020. All patients with ASD were informed of the advantages and disadvantages of the 3 ASD treatment options, and the family members of the patients chose treatment intentions according to the actual conditions. In this study, patients who underwent thoracic device closure were selected as study subjects. According to whether FTCA was administered, they were divided into group $\mathrm{F}$ (fast-track anesthesia, $\mathrm{n}=31$ ) and group $\mathrm{R}$ (routine anesthesia, $\mathrm{n}=30$ ).

Through preliminary experiments, it was calculated that the sample size needed to complete the study was 60 . Because this study was not prospective but rather retrospective, we tried our best to select matching patients, who accounted for only a small proportion of the patients in the same period. The anesthesiologist and the surgeon chose anesthesia according to their own experience and patient condition and received approval from parents. 
The inclusion criteria included transthoracic echocardiography (TTE) evidence of significant left-to-right shunts and isolated ASD, up to the indications of device closure, and no obvious contraindication to anesthesia and operation. Exclusion criteria included the following: (1) other cardiac abnormalities needing contemporaneous correction; (2) transfer to surgical repair under cardiopulmonary bypass; (3) severe pulmonary hypertension; (4) severe pneumonia and respiratory insufficiency or chronic cardiac, hepatic, or renal insufficiency; or (5) age $<1$ year. All selected patients completed a routine preoperative examination, and relevant data are collected in Table 1.

In group $\mathrm{F}$, all patients routinely fasted before surgery and were injected with $0.05 \mathrm{mg} / \mathrm{kg}$ midazolam intramuscularly before entering the operating room. Electrocardiography and peripheral blood oxygen saturation monitoring were performed after admission, and a $5 \%$ glucose solution was infused intravenously. Arterial blood pressure and central venous pressure were monitored by artery and subclavian vein puncture. Anesthesia induction included intravenous injection of $1.0 \mu \mathrm{g} / \mathrm{kg}$ remifentanil and $0.5 \mathrm{mg} / \mathrm{kg}$ cisatracurium besylate. Endotracheal intubation and mechanical ventilation were performed after muscle release. The ventilation mode was a pressure control mode. The nasopharyngeal temperature was monitored, and the body temperature was maintained at $>36.5^{\circ} \mathrm{C}$. Arterial blood gas was analyzed to assess ventilation conditions and the internal environment. The anesthesia was maintained by intravenous pumping with 0.1 to $0.5 \mu \mathrm{g} / \mathrm{kg} /$ min remifentanil and inhalation with $2 \%$ to $3 \%$ sevoflurane while pumping with $0.5 \mu \mathrm{g} / \mathrm{kg} / \mathrm{h}$ dexmedetomidine until the tracheal tube was removed.

The preparation in group $\mathrm{R}$ was the same as in group $\mathrm{F}$. Induction of anesthesia consisted of intravenous injection of 1 $\mu \mathrm{g} / \mathrm{kg}$ sufentanil and $0.5 \mathrm{mg} / \mathrm{kg}$ cisatracurium besylate. Anesthesia maintenance used 0.1 to $1 \mu \mathrm{g} / \mathrm{kg} / \mathrm{h}$ sufentanil by intravenous pumping and $2 \%$ to $3 \%$ sevoflurane by inhalation.

Postoperative analgesia and sedation management were the same in both groups. The patient-controlled analgesia method was used to perform intravenous analgesia for all patients after extubation. A standard protocol for $2.0 \mu \mathrm{g} / \mathrm{kg}$ sufentanil and $0.2 \mathrm{mg} / \mathrm{kg}$ tropisetron was used, and dosage was adjusted or stopped according to the patient's condition, determined by a unified ICU physician team.
A transesophageal echocardiography probe was placed into the patient's esophagus to measure the ASD diameter. A 2- to 3 -cm incision was made through the fourth intercostal space of the right sternum, and the rib spreader was placed. The pericardium was incised and suspended, and $1 \mathrm{mg} / \mathrm{kg}$ heparin was given intravenously. Continuous TTE guided the puncture to establish the right atrium/ASD/left atrium transport track and delivered and released the occluder. Care was taken that the occluder's position fitted well, with no residual shunt, no obstruction of the venae cavae or coronary sinus, and no significant effect on heart valve function and that electrocardiography indicated no serious atrioventricular block; the occluder was then released. The chest was closed routinely [Huang 2019; Chen 2012]. After the operation, the patient was sent to the ICU for further monitoring and treatment.

Data for statistical analysis were collected, including (1) general information before surgery; (2) perioperative hemodynamic index containing mean arterial blood pressure and heart rate before and after induction of general anesthesia, skin incision, thoracotomy, chest closure, skin suture, and extubation; (3) pain behavior scale (face, legs, activity, cry, consolability [FLACC] score) assessed at 1, 4, 8, and 24 hours after extubation; scores ranged from 0 to 10 , with higher score indicating more severe pain [Voepel-Lewis 2002]; and (4) Ramsay Sedation Scale (RSS) score (level 1, fidgety; level 2, peaceful cooperation; level 3, response to instruction; level 4 , lethargy, sensitive to light brow tap or intense sound stimulation; level 5, lethargy, slow response to light brow tap or intense voice stimulation; and level 6 , no response to light brow tap or intense sound stimulation) [Martinez 2002]; (5) duration of mechanical ventilation and length of ICU and hospital stays.

Continuous data all followed normal distribution by normal test and were statistically analyzed by independent sample $t$ test; $\chi^{2}$ test was used to compare the rate between the 2 groups. A $P$ value $<.05$ was defined as statistically significant.

\section{RESULTS}

Device closure of ASD was completed in all patients. Tables 1 and 2 show that there was no statistically significant

Table 1. General Preoperative Conditions of Patients*

\begin{tabular}{lccc}
\hline Characteristic & Group $F(n=31)$ & Group R $(\mathrm{n}=30)$ & $P$ value \\
\hline Age $(\mathrm{y})$ & $3.9 \pm 1.8$ & $4.5 \pm 1.9$ & .20 \\
Sex (M/F) & $13 / 18$ & $14 / 16$ & .75 \\
Body weight (kg) & $15.3 \pm 4.0$ & $17.2 \pm 4.8$ & .10 \\
ASD size (mm) & $18.3 \pm 5.0$ & $17.1 \pm 6.2$ & .80 \\
Pulmonary arterial pressure (mmHg) & $30.3 \pm 4.6$ & $30.8 \pm 5.5$ & .82 \\
Ejection fraction (\%) & $61.2 \pm 4.5$ & $60.5 \pm 5.0$ & .75 \\
\hline
\end{tabular}

*Data are mean \pm standard deviation. 
Table 2. Intraoperative Mean Arterial Blood Pressure (MAP) and Heart Rate (HR) between the 2 Groups*

\begin{tabular}{lccc}
\hline Measurement & Group $F(n=31)$ & Group R $(\mathrm{n}=30)$ & $P$ value \\
\hline After intubation MAP (mmHg) & $80.4 \pm 3.3$ & $78.1 \pm 3.3$ & .71 \\
After intubation HR (beats/min) & $83.3 \pm 3.5$ & $81.6 \pm 2.7$ & .83 \\
Skin incision MAP (mmHg) & $81.7 \pm 2.9$ & $79.2 \pm 2.9$ & .74 \\
Skin incision HR (beats/min) & $86.3 \pm 3.4$ & $84.0 \pm 2.4$ & .85 \\
Thoracotomy MAP (mmHg) & $83.0 \pm 2.5$ & $80.4 \pm 3.0$ & .69 \\
Thoracotomy HR (beats/min) & $88.5 \pm 3.6$ & $85.1 \pm 1.9$ & .65 \\
Close the chest MAP (mmHg) & $82.5 \pm 2.8$ & $79.3 \pm 2.9$ & .64 \\
Close the chest HR (beats/min) & $87.2 \pm 3.4$ & $84.0 \pm 1.8$ & .75 \\
Skin suture MAP (mmHg) & $81.8 \pm 2.6$ & $78.5 \pm 3.1$ & .72 \\
Skin suture HR (beats/min) & $86.0 \pm 3.2$ & $83.6 \pm 1.7$ & .83 \\
Extubation MAP (mmHg) & $81.6 \pm 3.0$ & $78.5 \pm 3.5$ & .76 \\
Extubation HR (beats/min) & $85.7 \pm 3.2$ & $83.5 \pm 2.2$ & .68 \\
\hline
\end{tabular}

*Data are mean \pm standard deviation.

Table 3. Pain Score (FLACC Score) at Each Time Point Between the 2 Groups*

\begin{tabular}{lccc}
\hline Score & Group $F(n=31)$ & Group $R(n=30)$ & $P$ value \\
\hline FLACC after extubation & & & .82 \\
$1 \mathrm{~h}$ & $0.2 \pm 0.5$ & $0.2 \pm 0.5$ & .25 \\
$4 \mathrm{~h}$ & $0.4 \pm 0.6$ & $0.3 \pm 0.5$ & .50 \\
$8 \mathrm{~h}$ & $1.4 \pm 0.5$ & $1.3 \pm 0.5$ & .76 \\
$24 \mathrm{~h}$ & $2.2 \pm 0.6$ & $2.3 \pm 0.5$ & .00 \\
RSS after extubation & & & .00 \\
$1 \mathrm{~h}$ & $3.4 \pm 0.5$ & $1.6 \pm 0.5$ & .01 \\
$8 \mathrm{~h}$ & $2.7 \pm 0.5$ & $1.7 \pm 0.5$ & .01 \\
$24 \mathrm{~h}$ & $2.1 \pm 0.4$ & $2.1 \pm 0.5$ & $2.2 \pm 0.5$ \\
\hline
\end{tabular}

*Data are mean \pm standard deviation.

Table 4. Postoperative Clinical Data between the 2 Groups*

\begin{tabular}{lccc}
\hline Factor & Group $F(n=31)$ & Group $(\mathrm{n}=30)$ & $P$ value \\
\hline Mechanical ventilation duration $(\mathrm{h})$ & $0.2 \pm 0.1$ & $1.2 \pm 0.4$ & .00 \\
Length of ICU stay $(\mathrm{h})$ & $1.6 \pm 0.2$ & $3.4 \pm 0.2$ & .00 \\
Length of hospital stay (d) & $4.8 \pm 0.4$ & $4.7 \pm 1.0$ & .65 \\
\hline
\end{tabular}

*Data are mean \pm standard deviation. 
difference in general preoperative conditions between the 2 groups $(P>.05)$. As shown in Table 2 , there was no statistically significant difference in perioperative hemodynamic indexes between the 2 groups $(P>.05)$. Table 3 shows no statistically significant difference between the 2 groups in pain scores $1,4,8$, or 24 hours after extubation $(P>.05)$. However, the patients' postoperative sedation scores in group $F$ were significantly higher than those in group R 1 and 4 hours after extubation $(P<.05)$ (Table 3$)$. Table 4 shows that the duration of mechanical ventilation and the length of ICU stay in group $\mathrm{F}$ was significantly shorter than in group $\mathrm{R}(P<.05)$, but there was no statistical difference in length of hospital stay between the groups. No severe complications associated with device closure or anesthesia occurred (retracheal intubation, laryngeal edema, airway obstruction, dislodgement of the device, etc).

\section{DISCUSSION}

ASD is one of the most common congenital heart diseases [Dickinson 1981]. Conventional surgical repair under cardiopulmonary bypass has broad indications and clear results. There is, however, a certain incidence of postoperative thoracic deformity; the incision scar affects aesthetics; and the hospital stay is relatively extended [Pastorek 1994]. In recent years, with the development of minimally invasive concepts and technology, the treatment of ASD has also developed in a minimally invasive direction. Percutaneous device closure of ASD has become an alternative treatment option. It has the advantages of no incision, no cardiopulmonary bypass, quick recovery, and short hospital stay [Sun 2010]. But the technique has some disadvantages, such as peripheral vascular trauma, radiation exposure, expensive equipment, a long conveying track, and a relatively complicated operation [Valente 2007]. Transthoracic closure of ASD with a small incision avoids some of these disadvantages. It is a cross between the 2 treatments and has the advantages of low trauma, a short conveying track, and intuitive operation through TTE/transesophageal guidance. If device closure fails, it can directly convert to surgical repair. It has been gradually recognized and is now widely used in China [Zhu 2013].

The development of minimally invasive cardiac surgery also necessitates higher requirements for corresponding anesthesia methods. FTCA mainly adjusts the use of appropriate anesthetic drugs to inprove the depth of anesthesia and meet surgery requirements. It can also help early recovery of postoperative spontaneous respiration, shorten mechanical ventilation duration and length of ICU stay, and reduce postoperative complications. Most studies have shown that FTCA is feasible and safe in pediatric cardiac surgery [Alghamdi 2010; Groesdonk 2010]. Harris et al [2014] found in a study of 613 children with congenital heart disease that $89 \%$ underwent FTCA, which reduced postoperative complications and length of ICU and postoperative hospital stays. Groesdonk et al [2010] followed up cardiac surgery cases under FTCA in their heart center and found that ultra-short-acting opioids for FTCA did not cause intraoperative knowledge and are considered safe and effective. The combination of FTCA and transthoracic device closure of ASD in children will enable patients to recover faster, reduce complications, and improve utilization of medical resources.

In traditional cardiac anesthesia, high-dose and long-acting opioids are used, leading to suppression of postoperative respiratory function and prolonged mechanical ventilation, thus increasing postoperative complications and length of hospital stay. Sufentanil is a highly fat-soluble opioid with a high binding rate of plasma protein and a clearance half-life of $150 \mathrm{~min}$. It is effective quickly and has the most potent analgesic effect, 10 times that of fentanyl. Postoperative resuscitation and extubation times are delayed with increased doses. It has little effect on the cardiovascular system or hemodynamics [Deshpande 2009]. As a new short-acting opioid agonist, remifentanil has an immediate effect, and spontaneous breathing can resume 3 to 5 minutes after stopping infusion. It is mainly hydrolyzed by nonspecific esterase in blood and tissues. It has a potent analgesic effect comparable to that of fentanyl, stable intraoperative hemodynamics, and good controllability [Friesen 2003; Engoren 2001]. However, studies have shown that the pain score with remifentanil was higher than that with sufentanil 1 hour after surgery [Lison 2007]. The average postoperative pain score of children with early extubation was $>5$ points, and inadequate analgesia was the most important reason for children's postoperative agitation [Reismann 2007]. Thus, hemodynamic stability is affected, and myocardial oxygen consumption is also increased. Simultaneously, incision pain affects cough and sputum excretion in children, increasing incidence of pulmonary infection and atelectasis. Therefore, adequate analgesia is necessary for children after FTCA, as it helps maintain the stability of hemodynamics, facilitates the recovery of respiratory function, and ensures that children pass the perioperative period smoothly.

Recently, there have been more and more studies on the application of dexmedetomidine in children [Ghai 2017; Carney 2017; Wei 2015]. Dexmedetomidine is a new type of $\alpha 2$ adrenal agonist with high efficiency, high selectivity, and sedative, analgesic, anti-anxiety, and anti-sympathetic nerve effects, and it can significantly reduce the amount of anesthesia in the perioperative period. It effectively alleviates the stress response, reduces postoperative pain and salivation, and has anti-chills, anti-nausea and diuretic, and other effects. Studies by Zhang et al [2015] and Soliman et al [2016] showed that dexmedetomidine could protect the myocardium and stabilize hemodynamics in cardiovascular surgery. Cheng et al [2015] found little variability in heart rate and blood pressure with dexmedetomidine infusion at 0.25 to $0.75 \mu \mathrm{g} /$ $\mathrm{kg} / \mathrm{h}$ during pediatric cardiac surgery anesthesia. Therefore, in the present study, patients in group $\mathrm{F}$ were treated with dexmedetomidine at an infusion dose of $0.5 \mu \mathrm{g} / \mathrm{kg} / \mathrm{h}$, and intraoperative hemodynamics were stable. Blaine et al [2007] also found that dexmedetomidine prevented agitation after sevoflurane anesthesia.

In our study, postoperative agitation of children in group $\mathrm{F}$ was statistically significantly less than that of group $R$, and the 
sedative effect was satisfactory. At the same time, this study also found that dexmedetomidine combined with remifentanil was more effective than sufentanil in inhibiting the stress response in the closure of transthoracic ASD in children. The pain scores at different time points showed that dexmedetomidine could prevent and improve remifentanil hyperalgesia. Although dexmedetomidine was continuously pumped until extubation in group $\mathrm{F}$, the results indicated that the duration of mechanical ventilation and length of ICU stay in group F were lower than those in group $\mathrm{R}$, which is consistent with a retrospective study of Nguyen et al [2016]. The unique properties of sedative and analgesia without respiratory depression make dexmedetomidine an ideal postoperative sedative for patients undergoing cardiac surgery, with the potential to reduce opioid use and anti-sympathetic effects. Hashemian et al [2017] drew similar conclusions, that continuous intravenous pumping of dexmedetomidine at $0.5 \mu \mathrm{g} / \mathrm{kg} / \mathrm{h}$ did not increase the duration of ICU patient resuscitation. Those characteristics of dexmedetomidine make it especially suitable for FTCA in transthoracic device closure of ASD in pediatric patients. In this study, there were no severe complications associated with fast-track anesthesia, which proves that remifentanil-based fast-track anesthesia combined with dexmedetomidine for transthoracic device closure of ASD in pediatric patients is safe.

This study was a single-center retrospective study. The small number of cases involved, the particular treatment approach, and the certain selective deviation in the research process might impact the results. For example, our study results showed that although there was a difference in the length of hospital stay between the 2 groups, the difference was not statistically significant, which differed from other studies [Huang 2019]. This might be due to the small sample size, the treatment philosophy of different hospitals, and patients' perceptions in different regions. However, we believe that these results have guiding significance for clinical practice. Future studies in prospective randomized controlled trials with larger sample sizes are needed to determine conclusions further.

\section{CONCLUSION}

Remifentanil combined with dexmedetomidine as FTCA for transthoracic device closure of ASD in pediatric patients can stabilize the hemodynamics in the perioperative period, reduce the incidence of postoperative agitation, and effectively reduce postoperative pain. Thus, duration of mechanical ventilation and ICU stay can be shortened, and children's recovery quality can be improved. The anesthesia model is worthy of clinical promotion.

\section{ACKNOWLEDGMENTS}

We gratefully acknowledge the participants' contributions, as well as Yi-Rong Zheng and all the anesthesiologists from Department of Cardiovascular Surgery, Union Hospital, Fujian Medical University.
REFERENCES

Alghamdi AA, Singh SK, Hamilton BC, Yadava M, Holtby H, Van Arsdell GS, Al-Radi OO. Early extubation after pediatric cardiac surgery: Systematic review, meta-analysis, and evidence-based recommendations. J Card Surg 2010;25:586-595.

Blaine Easley R, Brady KM, Tobias JD. Dexmedetomidine for the treatment of postanesthesia shivering in children. Paediatr Anaesth 2007;17:341-346.

Carney L, Kendrick J, Carr R. Safety and effectiveness of dexmedetomidine in the pediatric intensive care unit (SAD-PICU). Can J Hosp Pharm 2013;66:21-27.

Chen Q, Cao H, Zhang G-C, Chen L-W, Chen D-Z. Safety and feasibility of intra-operative device closure of atrial septal defect with transthoracic minimal invasion. Eur J Cardiothorac Surg 2012;41:121-125.

Cheng X, Zuo Y, Zhao Q, Gu E, Huang Y. Comparison of the effects of dexmedetomidine and propofol on hemodynamics and oxygen balance in children with complex congenital heart disease undergoing cardiac surgery. Congenit Heart Dis 2015;10:E123-E130.

Deshpande CM, Mohite SN, Kamdi P. Sufentanil vs fentanyl for fasttrack cardiac anaesthesia. Indian J Anaesth 2009;53:455-462.

Dickinson DF, Arnold R, Wilkinson JL. Congenital heart disease among 160,480 live-born children in Liverpool 1960 to 1969. Implications for surgical treatment. Br Heart J 1981;46:55-62.

Engoren M, Luther G, Fenn-Buderer N. A Comparison of fentanyl, sufentanil, and remifentanil for fast-track cardiac anesthesia. Anesth Analg 2001;93:859-864.

Friesen RH, Veit AS, Archibald DJ, Campanini RS. A comparison of remifentanil and fentanyl for fast track paediatric cardiac anaesthesia. Paediatr Anaesth 2003;13:122-125.

Ghai B, Jain K, Saxena AK, Bhatia N, Sodhi KS. Comparison of oral midazolam with intranasal dexmedetomidine premedication for children undergoing CT imaging: A randomized, double-blind, and controlled study. Paediatr Anaesth 2017;27:37-44.

Groesdonk HV, Pietzner J, Borger MA, Fassl J, Haentschel D, Paarmann $\mathrm{H}$, Ender J. The incidence of intraoperative awareness in cardiac surgery fast-track treatment. J Cardiothorac Vasc Anesth 2010;24:785-789.

Harris KC, Holowachuk S, Pitfield S, Sanatani S, Froese N, Potts JE, Gandhi SK. Should early extubation be the goal for children after congenital cardiac surgery? J Thorac Cardiovasc Surg 2014;148:2642-2647.

Hashemian M, Ahmadinejad M, Mohajerani SA, Mirkheshti A. Impact of dexmedetomidine on hemodynamic changes during and after coronary artery bypass grafting. Ann Card Anaesth 2017;20:152-157.

Huang Q, Lin L-Y, Lin X-Z. Comparison of remifentanil-based fasttrack and fentanyl-based routine cardiac anesthesia for intraoperative device closure of atrial septal defect (ASD) in pediatric patients. Med Sci Monit 2019;25:1187-1193.

Lison S, Schill M, Conzen P. Fast-track cardiac anesthesia: Efficacy and safety of remifentanil versus sufentanil. J Cardiothorac Vasc Anesth 2007;21:35-40.

Martinez JL, Sutters KA, Waite S, Davis J, Medina E, Montano N, et al. A comparison of oral diazepam versus midazolam, administered with intravenous meperidine, as premedication to sedation for pediatric endoscopy. J Pediatr Gastroenterol Nutr 2002;35:51-58.

Mittnacht AJ, Hollinger I. Fast-tracking in pediatric cardiac surgeryThe current standing. Ann Card Anaesth 2010;13:92-101. 
Nguyen J, Nacpil N. A Comparison between dexmedetomidine and propofol on extubation times in postoperative adult cardiac surgery patients: A systematic review protocol. JBI Database System Rev Implement Rep 2016;14:63-71.

Pastorek J, Allen H, Davis J. Current outcomes of surgical closure of secundum atrial septal defect. Am J Cardiol 1994;74:75-77.

Reismann M, von Kampen M, Laupichler B, Suempelmann R, Schmidt AI, Ure BM. Fast-track surgery in infants and children. J Pediatr Surg 2007;42:234-238.

Soliman R, Zohry G. The myocardial protective effect of dexmedetomidine in high-risk patients undergoing aortic vascular surgery. Ann Card Anaesth 2016;19:606-613.

Sun WF, Dong ZF, Gong K, Zhang GP, Cui T, Xia YD. Transcatheter closure with use of the SHSMA Occluder in 180 patients with congenital heart defects: Preliminary results. Tex Heart Inst J 2010;37:531-537.

Valente AM, Rhodes JF. Current indications and contraindications for transcatheter atrial septal defect and patent foramen ovale device closure.
Am Heart J 2007;153(4 suppl):81-84.

Verrier ED, Wright IH, Cochran RP, Spiess BD. Changes in cardiovascular surgical approaches to achieve early extubation. J Cardiothorac Vasc Anesth 1995;9(5 suppl 1):10-15.

Voepel-Lewis T, Merkel S, Tait AR, Trzcinka A, Malviya S. The reliability and validity of the face, legs, activity, cry, consolability observational tool as a measure of pain in children with cognitive impairment. Anesth Analg 2002;95:1224-1229.

Wei L, Deng X, Sui J, Wang L, Liu J. Dexmedetomidine improves intubating conditions without muscle relaxants in children after induction with propofol and remifentanil. Anesth Analg 2015;121:785-790.

Zhang X, Zhao X, Wang Y. Dexmedetomidine: A review of applications for cardiac surgery during perioperative period. J Anesth 2015;29:102-111.

Zhu P, Sun Y, Yang Q, Qiu F. Intraoperative device closure of atrial septal defects with minimal transthoracic invasion: A single-center experience. Tex Heart Inst J 2013;40:256-260. 